\title{
The H.E.S.S. Galactic Plane Survey
}

\author{
Emma de Oña-Wilhelmi for the H.E.S.S. collaboration \\ email: emma@mpi-hd.mpg.de
}

Max-Planck-Institute für Kernphysik, P.O. Box 103980, D 69029 Heidelberg, Germany

\begin{abstract}
The H.E.S.S. Galactic Plane Survey (GPS) has revealed a large number of Galactic Sources, including Pulsar Wind Nebulae (PWN), Supernova Remnants (SNRs), giant molecular clouds, star formation regions and compact binary systems, as well as a number of unidentified objects, or dark sources, for which no obvious counterparts at other wavelengths have yet been found. We will review the latest results from the GPS observations and discuss the most interesting cases.
\end{abstract}

Keywords. gamma rays: observations

The H.E.S.S. telescope is located in Namibia at $23^{\circ} 16^{\prime} 17^{\prime \prime} \mathrm{S} 16^{\circ} 29^{\prime} 58^{\prime \prime} \mathrm{E}$, at $1800 \mathrm{~m}$ above the sea level. H.E.S.S. is a system of four large (13 $\mathrm{m}$ diameter) imaging atmospheric Cherenkov telescopes, designed to detect gamma-rays in the very-high-energy (VHE; E > $100 \mathrm{GeV}$ ) domain. Each telescope has a mirror area of $107 \mathrm{~m}^{2}$ and a total field of view of $5^{\circ}$, well suited to the study of extended sources. The system works in a coincidence mode. Its angular resolution reaches $\sim 5^{\prime}$ per event and its sensitivity for a point-like source, is $2.0 \times 10^{-13} \mathrm{ph} \mathrm{cm}^{-2} \mathrm{~s}^{-1}(1 \%$ of the Crab Nebula flux above $1 \mathrm{TeV})$ for a $5 \sigma$ detection, within 25 hours observation time. The H.E.S.S. telescope has conducted an extension of the scan of the inner Galactic Plane Survey (GPS, Aharonian et al. (2005)), which was a major breakthrough in the Galactic field. This report can only give a brief overview; for details and further references, the reader is referred to the publications cited. The survey covers the as yet unexplored range in longitude between $\left[-85^{\circ}, 60^{\circ}\right]$ and $\left[-2.5^{\circ}, 2.5^{\circ}\right]$ in latitude. It has revealed more than two dozen new VHE sources, consisting of shell-type SNRs, PWN, X-ray binary systems, two putative young star clusters, as yet unidentified objects, the so-called dark sources, in which no obvious counterparts at other energy wavelengths are found (see e.g. Aharonian et al. (2006), Aharonian et al. (2008)). In the central 100 pc of the Milky Way diffuse emission is seen, and we are able to locate the Galactic Centre $\gamma$-ray source with a precision of $6^{\prime \prime}$, consistent with the black hole Sgr A*, but excluding the nearby remnant Sgr A East. The sources line up along the Galactic plane, with a rms spread in latitude of about $0.3^{\circ}$, consistent with the scale height of the distribution of molecular gas and with the width of the distribution of supernova remnants and pulsars. Their Galactic origin is confirmed by the fact that nearly all sources are extended, with rms sizes up to $0.3^{\circ}$. The official H.E.S.S. Source Catalogue can be found at www.mpi-hd.mpg.de/hfm/HESS/pages/home/sources and includes all of the VHE $\gamma$-ray sources which were detected by H.E.S.S. and subsequently published in refereed journals.

\section{References}

Aharonian, F. A. et al. (H. E. S. S. Collaboration) 2005, Science, 307, 1938.

Aharonian F. A. et al. (H. E. S. S. Collaboration) 2006, ApJ, 636, 777.

Aharonian F. A. et al. (H. E. S. S. Collaboration), 2008, A\&A, 477, 353. 